\title{
Ensemble algorithm for parametrized flow problems with energy stable open boundary conditions
}

\author{
Aziz Takhirov • Jiajia Waters
}

\begin{abstract}
We propose a novel ensemble calculation method for Navier-Stokes equations subject to various initial conditions, forcing terms and viscosity coefficients. We establish the stability of the scheme under the CFL condition that is same as the single viscosity coefficient case. Moreover, we extend the ensemble calculation method to problems with open boundary conditions, with provable energy stability.
\end{abstract}

Keywords Ensemble simulations · open boundary conditions · incompressible Navier-Stokes Equations

\section{Introduction}

We consider $J$ Navier-Stokes equations subject to perturbed initial conditions $u_{j}^{0}$, body forces $f_{j}$ and viscosity coefficients $\nu_{j}$ :

$$
\begin{aligned}
\partial_{t} u_{j}+u_{j} \cdot \nabla u_{j}-\nabla \cdot\left(\nu_{j}(x) \nabla u_{j}\right)+\nabla p_{j} & =f_{j}(x, t) \text { in } \Omega, \\
\nabla \cdot u_{j} & =0 \text { in } \Omega, \\
u_{j} & =0 \text { on } \partial \Omega, \\
u_{j}(x, 0) & =u_{j}^{0}(x) \text { in } \Omega,
\end{aligned}
$$

where $\Omega$ denotes the physical domain and $j=\overline{1, J}$. When the system (1)-(4) is solved by linearly implicit methods, the corresponding linear system matrix will depend on the ensemble member $j$, due to the nonlinear and diffusion terms, and thus must be assembled $J$ times. The advantage of the semi-implicit approach is obvious; one can pick the timestep solely based on accuracy considerations. However, in practical applications, such as sensitivity analysis of the scheme to problem parameters [1,2], reduced order modelling [3,4,5] and ensemble forecasting $[6,7], J$ tends to be quite large. Solving one system of the form (1)-(4) is challenging by itself, and the computational cost of obtaining accurate solutions of all ensembles members by this approach maybe prohibitive.

In the $\nu_{j}=\nu=$ const case, one alternative to a semi-implicit approach is to treat the nonlinear term fully explicitly, and thereby assemble a single linear system once for all. However, for high Re flows, this strategy induces a very restrictive timestep condition, and the computational cost of the scheme could exceed the cost of the semi-implicit approach. The computational cost increases even further on adaptively refined meshes.

\footnotetext{
A. Takhirov Canada.

E-mail: takhirov@ualberta.ca

J. Waters

Los Alamos National Laboratory, Los Alamos, NM 87545, USA.

E-mail: jwaters@lanl.gov
}

Department of Mathematical and Statistical Sciences, University of Alberta, Edmonton, AB, T6G 2G1, 
The first work for the efficient ensemble calculation was proposed in [9], which considered the $\nu_{j}=\nu=$ const case. The scheme was first order in time, and suitable for low Re number flows. The idea was later extended to higher order schemes and high Re flows in [12], [13], and [14]. The unifying idea of in all of these works is to split the advecting velocity in the nonlinear term into ensemble mean plus fluctuating part, make it explicit, and then treat the first nonlinear term semi-implicitly, while make the fluctuating part fully explicit. The energy stability then can be shown to hold under a timestep restriction involving the velocity fluctuations, which should not be as restrictive as the fully explicit approach.

The case of the multiple, constant viscosity coefficients has been recently addressed in [8]. Since $\nu_{j} \triangle u_{j}$ term is nonlinear with respect to ensemble member $j$, the splitting similar to the treatment of the nonlinearity was considered. Denoting the mean viscosity by $\bar{\nu}=\frac{1}{J} \sum_{j=1}^{J} \nu_{j}$, the following scheme was studied:

$$
\begin{aligned}
& \frac{u_{j}^{n+1}-u_{j}^{n}}{\Delta t}+\overline{u^{n}} \cdot \nabla u_{j}^{n+1}+\left(u_{j}^{n}-\overline{u^{n}}\right) \cdot \nabla u_{j}^{n}-\nu_{j} \triangle u_{j}^{n} \\
&-\bar{\nu} \triangle\left(u_{j}^{n+1}-u_{j}^{n}\right)+\nabla p_{j}^{n+1}=f_{j}\left(x, t_{n+1}\right) \text { in } \Omega, \\
& \nabla \cdot u_{j}^{n+1}=0 \text { in } \Omega .
\end{aligned}
$$

Although, the resulting linear system is independent of the ensemble member $j$, the stability of the scheme holds, besides a timestep restriction, under an additional assumption:

$$
\frac{\left|\nu_{j}-\bar{\nu}\right|}{\bar{\nu}} \leq \sqrt{\mu}, \text { for some } \mu \in[0,1) .
$$

One can easily construct an example where this condition would be violated. For example, assuming $J>2$, and that viscosities are numbered in the increasing order, no $\mu$ satisfying (7) exist, if one chooses $\nu_{J}>\frac{2}{J-2} \sum_{j=1}^{J-1} \nu_{j}$.

In this work, we consider a different treatment of the diffusive term which allows to avoid any restriction on the viscosity coefficients, cf. Theorem 7 . We also extend the ensemble scheme to problems with open boundaries. To this end, we decompose the boundary $\partial \Omega$ into Dirichlet boundary $\Gamma_{D}$ and open boundary $\Gamma_{N}$. We further partitition $\Gamma_{N}$ boundary into the outflow and backflow regions:

$$
\Gamma_{N}=\Gamma_{j, N}^{+} \cap \Gamma_{j, N}^{-},
$$

where

$$
\Gamma_{j, N}^{+}:=\left\{x \in \Gamma_{N}:\left(u_{j} \cdot \mathbf{n}\right)(x)>0\right\} \text { and } \Gamma_{j, N}^{-}:=\left\{x \in \Gamma_{N}:\left(u_{j} \cdot \mathbf{n}\right)(x) \leq 0\right\} .
$$

On $\Gamma_{N}$, we assume the following energy stable boundary condition:

$$
\left(-\nu_{j} \nabla u_{j}+p_{j} \mathrm{I}\right) \mathbf{n}=\frac{\left(u_{j} \cdot \mathbf{n}\right) u_{j}}{2}\left(\mathrm{H}\left(\mathrm{u}_{\mathrm{j}} \cdot \mathbf{n}\right)-1\right)+\mathrm{L} \partial_{t} u_{j}
$$

where $\mathbf{n}$ denotes the unit normal on the boundary, $\mathrm{H}$ is the Heaviside function and $\mathrm{L}$ is the characteristic length scale. One can recognize that (8), up to the the factor L, is same as the the convective-like open boundary condition proposed in [15]. Using L instead of the original constant $\frac{\nu}{U}$ allows us to obtain a stability bound with a favourable constant.

In this presentation, we restrict the analysis to the constant viscosity case. One important example of the non-constant viscosity occurs when the eddy viscosity hypothesis is applied for the ensemble of Re $\gg 1$ flows. The schemes we propose can be easily extended to this case as well, when combined with the nonlinear filter based stabilization method of [23].

This paper is arranged as follows. Section 2 contains the preliminaries and notations. Section 3 presents the weak formulations of the Algorithms. Sections 4 proves energy stability, and Section 5 contains numerical experiments. The last section gives a conclusion of the studies. 


\section{Preliminaries}

Given ensemble $g_{1}, \ldots, g_{J}$ of a quantity $g$, we define the fluctuation in $j$-th member as

$$
g_{j}^{\prime}=g_{j}-\bar{g},
$$

and its $l^{\infty}$ norm by

$$
g_{\infty}=\max _{1 \leq j \leq J} g_{j}
$$

The $L^{2}(\Omega)$ norm and inner product will be denoted by $\|\cdot\|$ and $(\cdot, \cdot)$, while the $L^{\infty}$ norm over a domain $\gamma$ will be denoted as $\|\cdot\|_{\infty, \gamma}$. For simplicity of the presentation, we assume no-slip boundary condition on $\Gamma_{D}$. In this setting, the appropriate velocity and pressure spaces are defined as

$$
X:=\left(H_{0}^{1}(\Omega)\right)^{d}, X_{D}:=\left\{v \in\left(H^{1}(\Omega)\right)^{d}: v=0 \text { on } \Gamma_{D}\right\}, Q:=L_{0}^{2}(\Omega) .
$$

We use as the norm on $X$ and $X_{D}$, the seminorm $\|\nabla v\|_{L^{2}}$. The space of divergence free functions is given by

$$
V:=\{v \in X:(\nabla \cdot v, q)=0 \quad \forall q \in Q\} .
$$

The dual spaces $X^{*}=H^{-1}(\Omega)$ and $X_{D}^{*}=H_{D}^{-1}(\Omega)$ are equipped with norms

$$
\|f\|_{-1}=\sup _{v \in X} \frac{\langle f, v\rangle}{\|\nabla v\|}, \text { and }\|f\|_{-1, D}=\sup _{v \in X_{D}} \frac{\langle f, v\rangle}{\|\nabla v\|},
$$

where $\langle\cdot, \cdot\rangle$ refers to duality pairings.

We denote conforming velocity, pressure finite element spaces based on an edge to edge triangulations (tetrahedralizations) of $\Omega$ (with maximum element diameter $h$ ) by

$$
X_{h} \subset X\left(X_{D, h} \subset X_{D}\right), Q_{h} \subset Q .
$$

We assume that $X_{h}, Q_{h}$ satisfy the usual inf-sup stability condition [22]. The space of discrete, weakly divergence free functions is given by

$$
V_{h}:=\left\{v_{h} \in X_{h}:\left(\nabla \cdot v_{h}, q_{h}\right)=0, \quad \forall q_{h} \in Q_{h}\right\} .
$$

The trilinear term is denoted by

$$
b(u, v, w)=(u \cdot \nabla v, w) .
$$

In discrete setting, $b(u, v, w)$ must be skew-symmetrized to ensure energy stability of the scheme. There are multiple variations discussed in the literature, cf. [21] for one recent result. In our analysis and numerical tests, we will make use of the following skew-symmetrization of $b(u, v, w)$ :

$$
b_{1}(u, v, w):=b(u, v, w)+\frac{1}{2}(\nabla \cdot u, w \cdot v)
$$

For the implementation of the open boundary conditions, we introduce another trilinear term:

$$
b_{2}(u, v, w):=-\frac{1}{2}\left((u \cdot n) \Theta_{0}(u \cdot \mathbf{n}), v \cdot w\right)_{\Gamma_{N}}
$$

where

$$
\Theta_{0}(u \cdot \mathbf{n})=\frac{1}{2}\left(1-\tanh \frac{u \cdot \mathbf{n}}{\varepsilon U_{0}}\right) \simeq \mathrm{H}(u \cdot \mathbf{n})-1, \text { and } \Theta_{1}(u \cdot \mathbf{n})=1-\Theta_{0}(u \cdot \mathbf{n}),
$$

$\varepsilon \ll 1$ and $U_{0}$ is a reference speed. We note that for the problems with open boundaries, $b_{1}(\cdot, \cdot, \cdot)$ is more accurate than another commonly used skew-symmetrization

$$
b_{3}(u, v, w):=\frac{1}{2}(b(u, v, w)-b(u, w, v))
$$


in a sense that, if $u \in X_{D}$, divergence free and $v \in X_{D}$, then

$$
b(u, v, v)=b_{1}(u, v, v)=\int_{\Gamma_{N}} \frac{u \cdot \mathbf{n}}{2}|v|^{2}, \text { while } b_{3}(u, v, v)=0 .
$$

We will also make use of the Gronwall's Lemma.

Lemma 1 (Gronwall's inequality.) Assume $\left\{a_{n}\right\},\left\{b_{n}\right\}$ are nonnegative sequences, $c>0$ and

$$
a_{n} \leq c+\sum_{0 \leq k<n} a_{k} b_{k} \text { for } n \geq 0
$$

Then

$$
a_{n} \leq c \prod_{0 \leq k<n}\left(1+b_{k}\right) \leq c \exp \left(\sum_{0 \leq k<n} b_{k}\right) \text { for } n \geq 0 \text {. }
$$

\section{Numerical schemes}

\subsection{First order schemes}

For the case of the pure Dirichlet boundary condition, our first order algorithm approximating (1)-(4) takes the following form.

Algorithm 1 Given $J$ initial velocities $u_{j}^{0} \in V$, forcing terms $f_{j} \in H^{-1}(\Omega)$ and viscosities $\nu_{j}$, a time step $\Delta t>0$, find $\left(u_{j, h}^{n+1}, p_{j, h}^{n+1}\right) \in\left(X_{h}, Q_{h}\right), n=0,1, \ldots, N-1$, satisfying

$$
\begin{aligned}
\frac{\left(u_{j, h}^{n+1}-u_{j, h}^{n}, v_{h}\right)}{\Delta t}+b_{1}\left(\overline{u_{h}^{n}}, u_{j, h}^{n+1}, v_{h}\right)+b_{1}\left(u_{j, h}^{n^{\prime}}, u_{j, h}^{n}, v_{h}\right) & -\left(p_{j, h}^{n+1}, \nabla \cdot v_{h}\right) \\
+\nu_{j}\left(\nabla u_{j, h}^{n}, \nabla v_{h}\right)+\nu_{\infty}\left(\nabla\left(u_{j, h}^{n+1}-u_{j, h}^{n}\right), \nabla v_{h}\right) & =\left\langle f_{j}\left(t^{n+1}\right), v_{h}\right\rangle, \\
\left(\nabla \cdot u_{j, h}^{n+1}, q_{h}\right) & =0,
\end{aligned}
$$

for all $v_{h} \in X_{h}$ and $q_{h} \in Q_{h}$.

Now we turn to the case with outflow boundary. To this end, we first derive the weak formulation of the continuous system (1)-(4) under the following perturbation of the open boundary condition (8) for the ensemble case:

$$
\left(-\nu_{j} \nabla u_{j}-\Delta t \nu_{\infty} \nabla \partial_{t} u_{j}+p_{j} \mathrm{I}\right) \mathbf{n}=\frac{\left(u_{j} \cdot \mathbf{n}\right) u_{j}}{2} \Theta_{0}\left(u_{j} \cdot \mathbf{n}\right)+\mathrm{L} \partial_{t} u_{j}
$$

Then the weak form takes following form:

$$
\begin{aligned}
\left(\partial_{t} u_{j}, v\right)+\mathrm{L}\left(\partial_{t} u_{j}, v\right)_{\Gamma_{N}}+b_{1}\left(u_{j}, u_{j}, v\right)+b_{2}\left(u_{j}, u_{j}, v\right) & +\nu_{j}\left(\nabla u_{j}, \nabla v\right) \\
+\Delta t \nu_{\infty}\left(\nabla \partial_{t} u_{j}, \nabla v\right)-\left(p_{j}, \nabla \cdot v\right) & =\left\langle f_{j}, v\right\rangle \\
\left(\nabla \cdot u_{j}, q_{h}\right) & =0
\end{aligned}
$$

To derive the scheme for the ensemble calculation, we treat both nonlinear term and the viscous term as in Algorithm 1:

Algorithm 2 Given $J$ initial velocities $u_{j}^{0} \in V$, forcing terms $f_{j} \in X_{D}^{*}$ and viscosities $\nu_{j}, a$ time step $\Delta t>0$, find $\left(u_{j, h}^{n+1}, p_{j, h}^{n+1}\right) \in\left(X_{D, h}, Q_{h}\right), n=0,1, \ldots, N-1$ satisfying

$$
\begin{aligned}
\frac{\left(u_{j, h}^{n+1}-u_{j, h}^{n}, v_{h}\right)+\mathrm{L}\left(u_{j, h}^{n+1}-u_{j, h}^{n}, v_{h}\right)_{\Gamma_{N}}+\sum_{i=1}^{2}\left(b_{i}\left(\overline{u_{h}^{n}}, u_{j, h}^{n+1}, v_{h}\right)\right.}{\Delta t} & \left.b_{i}\left(u_{j, h}^{n^{\prime},}, u_{j, h}^{n}, v_{h}\right)\right) \\
+\nu_{j}\left(\nabla u_{j, h}^{n}, \nabla v_{h}\right)+\nu_{\infty}\left(\nabla\left(u_{j, h}^{n+1}-u_{j, h}^{n}\right), \nabla v_{h}\right)-\left(p_{j, h}^{n+1}, \nabla \cdot v_{h}\right) & =\left\langle f_{j}^{n+1}, v_{h}\right\rangle, \\
\left(\nabla \cdot u_{j, h}^{n+1}, q_{h}\right) & =0
\end{aligned}
$$

for all $v_{h} \in X_{D, h}$ and $q_{h} \in Q_{h}$. 
Another scheme can be derived by replacing $\sum_{i=1}^{2} b_{i}\left(u_{j, h}^{n^{\prime}}, u_{j, h}^{n}, v_{h}\right)$ with $b_{3}\left(u_{j, h}^{n^{\prime}}, u_{j, h}^{n}, v_{h}\right)$ :

Algorithm 3 Given $J$ initial velocities $u_{j}^{0} \in V$, forcing terms $f_{j} \in X_{D}^{*}$ and viscosities $\nu_{j}, a$ time step $\Delta t>0$, find $\left(u_{j, h}^{n+1}, p_{j, h}^{n+1}\right) \in\left(X_{D, h}, Q_{h}\right), n=0,1, \ldots, N-1$ satisfying

$$
\begin{aligned}
\frac{\left(u_{j, h}^{n+1}-u_{j, h}^{n}, v_{h}\right)}{\Delta t}+\sum_{i=1}^{2} b_{i}\left(\overline{u_{h}^{n}}, u_{j, h}^{n+1}, v_{h}\right)+b_{3}\left(u_{j, h}^{n^{\prime}}, u_{j, h}^{n}, v_{h}\right) & +\nu_{j}\left(\nabla u_{j, h}^{n}, \nabla v_{h}\right) \\
+\nu_{\infty}\left(\nabla\left(u_{j, h}^{n+1}-u_{j, h}^{n}\right), \nabla v_{h}\right)-\left(p_{j, h}^{n+1}, \nabla \cdot v_{h}\right) & =\left\langle f_{j}^{n+1}, v_{h}\right\rangle, \\
\left(\nabla \cdot u_{j, h}^{n+1}, q_{h}\right) & =0,
\end{aligned}
$$

for all $v_{h} \in X_{D, h}$ and $q_{h} \in Q_{h}$.

Note that we set $\mathrm{L}=0$ in this case, as this term is not necessary for proving the stability in this case.

\subsection{Second order schemes}

Denoting $E_{j, h}^{n}:=2 u_{j, h}^{n}-u_{j, h}^{n-1}$, we immediately obtain the second order extensions of the Algorithms 1-3.

Algorithm 4 Given $J$ initial velocities $u_{j}^{0} \in V$, forcing terms $f_{j} \in H^{-1}(\Omega)$ and viscosities $\nu_{j}$, a time step $\Delta t>0$, find $\left(u_{j, h}^{n+1}, p_{j, h}^{n+1}\right) \in\left(X_{h}, Q_{h}\right), n=0,1, \ldots, N-1$, satisfying

$$
\begin{aligned}
\frac{\left(3 u_{j, h}^{n+1}-4 u_{j, h}^{n}+u_{j, h}^{n-1}, v_{h}\right)}{2 \Delta t}+b_{1}\left(\overline{E_{h}^{n}}, u_{j, h}^{n+1}, v_{h}\right)+b_{1}\left(E_{j, h}^{n^{\prime}}, E_{j, h}^{n}, v_{h}\right) & =\left(p_{j, h}^{n+1}, \nabla \cdot v_{h}\right) \\
+\nu_{j}\left(\nabla E_{j, h}^{n}, \nabla v_{h}\right)+\nu_{\infty}\left(\nabla\left(u_{j, h}^{n+1}-E_{j, h}^{n}\right), \nabla v_{h}\right) & =\left\langle f_{j}\left(t^{n+1}\right), v_{h}\right\rangle \\
\left(\nabla \cdot u_{j, h}^{n+1}, q_{h}\right) & =0
\end{aligned}
$$

for all $v_{h} \in X_{h}$ and $q_{h} \in Q_{h}$.

Algorithm 5 Given $J$ initial velocities $u_{j}^{0} \in V$, forcing terms $f_{j} \in X_{D}^{*}$ and viscosities $\nu_{j}, a$ time step $\Delta t>0$, find $\left(u_{j, h}^{n+1}, p_{j, h}^{n+1}\right) \in\left(X_{D, h}, Q_{h}\right), n=0,1, \ldots, N-1$ satisfying

$$
\begin{aligned}
\frac{\left(3 u_{j, h}^{n+1}-4 u_{j, h}^{n}+\right.}{\left.u_{j, h}^{n-1}, v_{h}\right)+\mathrm{L}\left(3 u_{j, h}^{n+1}-4 u_{j, h}^{n}+u_{j, h}^{n-1}, v_{h}\right)_{\Gamma_{N}}} & 2 \Delta t \\
+ & \sum_{i=1}^{2}\left(b_{i}\left(\overline{E_{h}^{n}}, u_{j, h}^{n+1}, v_{h}\right)+b_{i}\left(E_{j, h}^{n^{\prime}}, E_{j, h}^{n}, v_{h}\right)\right)+\nu_{j}\left(\nabla E_{j, h}^{n}, \nabla v_{h}\right) \\
+\nu_{\infty}\left(\nabla\left(u_{j, h}^{n+1}-E_{j, h}^{n}\right), \nabla v_{h}\right)-\left(p_{j, h}^{n+1}, \nabla \cdot v_{h}\right) & =\left\langle f_{j}^{n+1}, v_{h}\right\rangle, \\
\left(\nabla \cdot u_{j, h}^{n+1}, q_{h}\right) & =0,
\end{aligned}
$$

for all $v_{h} \in X_{D, h}$ and $q_{h} \in Q_{h}$.

Algorithm 6 Given $J$ initial velocities $u_{j}^{0} \in V$, forcing terms $f_{j} \in X_{D}^{*}$ and viscosities $\nu_{j}, a$ time step $\Delta t>0$, find $\left(u_{j, h}^{n+1}, p_{j, h}^{n+1}\right) \in\left(X_{D, h}, Q_{h}\right), n=0,1, \ldots, N-1$ satisfying

$$
\begin{aligned}
& \frac{\left(3 u_{j, h}^{n+1}-4 u_{j, h}^{n}+u_{j, h}^{n-1}, v_{h}\right)}{2 \Delta t}+\sum_{i=1}^{2} b_{i}\left(\overline{E_{h}^{n}}, u_{j, h}^{n+1}, v_{h}\right)+b_{3}\left(E_{j, h}^{n^{\prime}}, E_{j, h}^{n}, v_{h}\right)+\nu_{j}\left(\nabla E_{j, h}^{n}, \nabla v_{h}\right) \\
& +\nu_{\infty}\left(\nabla\left(u_{j, h}^{n+1}-E_{j, h}^{n}\right), \nabla v_{h}\right)-\left(p_{j, h}^{n+1}, \nabla \cdot v_{h}\right)=\left\langle f_{j}^{n+1}, v_{h}\right\rangle, \\
& \left(\nabla \cdot u_{j, h}^{n+1}, q_{h}\right)=0
\end{aligned}
$$

for all $v_{h} \in X_{D, h}$ and $q_{h} \in Q_{h}$. 
All the Algorithms 1-6 give rise to a matrices that are independent of the ensemble member, and thus require that only a single coefficient matrix is stored along with $J$ right-hand sides at each time step. The resulting linear systems could be solved efficiently using solvers for systems with multiple right-hand sides, cf. [19,20].

\section{Theoretical resutls}

In this section we prove the stability results for the numerical schemes. We are able to show energy stability under timestep conditions for the first order schemes. However, we were not able to prove the stability results for the second order methods without restrictions on the viscosities, similar to (7), or with very pessimistic exponential bound. The main difficulty here is the fact that $\left(\nabla \partial_{t t} u, \nabla u\right)$ term is neither purely energy contributing term nor its purely dissipative. Nonetheless, the numerical experiments show that the numerical schemes are energy stable under the same CFL conditions as those of first order schemes.

\subsection{Stability with homogeneous Dirichlet boundary conditions}

In this subsection, we consider the $\Gamma_{N}=\emptyset$ case, and we will establish the stability under a time step condition:

$$
\frac{\Delta t}{\nu_{j}}\left(\left\|u_{j, h}^{n^{\prime}}\right\|_{\infty}+\frac{\operatorname{diam}(\Omega)}{d}\left\|\nabla \cdot u_{j, h}^{n^{\prime}}\right\|_{\infty}\right)^{2} \leq 1, \forall j=1, \ldots, J,
$$

for the Algorithm 1.

\section{Theorem 7 Let}

$$
E n_{j}^{n}:=\frac{\left\|u_{j, h}^{n}\right\|^{2}}{2}+\Delta t \frac{\nu_{\infty}}{2}\left\|\nabla u_{j, h}^{n}\right\|^{2} .
$$

If for each time step $n \geq 1$, the condition (24) holds, then the solutions to Algorithm 1 satisfy

$$
E n_{j}^{N} \leq E n_{j}^{0}+\sum_{n=0}^{N-1} \frac{\Delta t}{2 \nu_{j}}\left\|f_{j}\left(t^{n+1}\right)\right\|_{-1}^{2} \text { for all } j .
$$

Proof Choose $v_{h}=u_{j, h}^{n+1}$ in (9), $q_{h}=p_{j, h}^{n+1}$ in (10) and add them to get

$$
\begin{aligned}
\frac{\left(u_{j, h}^{n+1}-u_{j, h}^{n}, u_{j, h}^{n+1}\right)}{\Delta t} & +b_{1}\left(u_{j, h}^{n^{\prime}}, u_{j, h}^{n}, u_{j, h}^{n+1}\right)+\nu_{\infty}\left\|\nabla u_{j, h}^{n+1}\right\|^{2} \\
+\left(\nu_{j}-\nu_{\infty}\right)\left(\nabla u_{j, h}^{n}, \nabla u_{j, h}^{n+1}\right) & =\left\langle f_{j}\left(t^{n+1}\right), u_{j, h}^{n+1}\right\rangle .
\end{aligned}
$$

Applying the polarization identity gives

$$
\begin{aligned}
\frac{\left\|u_{j, h}^{n+1}\right\|^{2}-\left\|u_{j, h}^{n}\right\|^{2}+\| u_{j, h}^{n+1}-}{2 \Delta t} u_{j, h}^{n} \|^{2} & +\nu_{\infty}\left\|\nabla u_{j, h}^{n+1}\right\|^{2}=\left\langle f_{j}\left(t^{n+1}\right), u_{j, h}^{n+1}\right\rangle \\
-b_{1}\left(u_{j, h}^{n^{\prime}}, u_{j, h}^{n}, u_{j, h}^{n+1}\right) & +\left(\nu_{\infty}-\nu_{j}\right)\left(\nabla u_{j, h}^{n}, \nabla u_{j, h}^{n+1}\right) .
\end{aligned}
$$

It remains to bound the terms on the right hand side. Using the generalized Hölder's and Young's inequalities, we obtain

$$
\begin{aligned}
b_{1}\left(u_{j, h}^{n^{\prime}}, u_{j, h}^{n}, u_{j, h}^{n+1}\right) & =b_{1}\left(u_{j, h}^{n^{\prime}}, u_{j, h}^{n}, u_{j, h}^{n+1}-u_{j, h}^{n}\right) \\
& \leq\left(\left\|u_{j, h}^{n^{\prime}}\right\|_{\infty}\left\|\nabla u_{j, h}^{n}\right\|+\frac{\left\|\nabla \cdot u_{j, h}^{n^{\prime}}\right\|_{\infty}}{2}\left\|u_{j, h}^{n}\right\|\right)\left\|u_{j, h}^{n+1}-u_{j, h}^{n}\right\| \\
& \leq \frac{\left\|u_{j, h}^{n+1}-u_{j, h}^{n}\right\|^{2}}{2 \Delta t}+\frac{\Delta t}{2}\left(\left\|u_{j, h}^{n^{\prime}}\right\|_{\infty}\left\|\nabla u_{j, h}^{n}\right\|+\frac{\left\|\nabla \cdot u_{j, h}^{n^{\prime}}\right\|_{\infty}}{2}\left\|u_{j, h}^{n}\right\|\right)^{2} \\
& \leq \frac{\left\|u_{j, h}^{n+1}-u_{j, h}^{n}\right\|^{2}}{2 \Delta t}+\frac{\Delta t}{2}\left\|\nabla u_{j, h}^{n}\right\|^{2}\left(\left\|u_{j, h}^{n^{\prime}}\right\|_{\infty}+\frac{\left\|\nabla \cdot u_{j, h}^{n^{\prime}}\right\|_{\infty}}{2} C_{p}\right)^{2},
\end{aligned}
$$


where $C_{p}=\frac{2 \operatorname{diam}(\Omega)}{d}$ is the Poincaré's constant ([16, pg. 22]). Further, we get

$$
\left(\nu_{\infty}-\nu_{j}\right)\left(\nabla u_{j, h}^{n}, \nabla u_{j, h}^{n+1}\right) \leq \frac{\nu_{\infty}-\nu_{j}}{2}\left\|\nabla u_{j, h}^{n}\right\|^{2}+\frac{\nu_{\infty}-\nu_{j}}{2}\left\|\nabla u_{j, h}^{n+1}\right\|^{2},
$$

and

$$
\left\langle f_{j}\left(t^{n+1}\right), u_{j, h}^{n+1}\right\rangle \leq \frac{1}{2 \nu_{j}}\left\|f_{j}\left(t^{n+1}\right)\right\|_{-1}^{2}+\frac{\nu_{j}}{2}\left\|\nabla u_{j, h}^{n+1}\right\|^{2} .
$$

Combining (27) - (30) yields

$$
\begin{aligned}
\frac{\left\|u_{j, h}^{n+1}\right\|^{2}-\left\|u_{j, h}^{n}\right\|^{2}}{2 \Delta t} & +\frac{\nu_{\infty}}{2}\left[\left\|\nabla u_{j, h}^{n+1}\right\|^{2}-\left\|\nabla u_{j, h}^{n}\right\|^{2}\right] \\
& +\left[\frac{\nu_{j}}{2}-\frac{\Delta t}{2}\left(\left\|u_{j, h}^{n^{\prime}}\right\|_{\infty}+\frac{\left\|\nabla \cdot u_{j, h}^{n^{\prime}}\right\|_{\infty}}{2} C_{p}\right)^{2}\right]\left\|\nabla u_{j, h}^{n}\right\|^{2} \\
& \leq \frac{1}{2 \nu_{j}}\left\|f_{j}\left(t^{n+1}\right)\right\|_{-1}^{2} .
\end{aligned}
$$

Under the CFL condition (24), the last term on the left hand side of (31) is nonnegative and summing over the timesteps completes the proof.

Remark 1 We can obtain an improved stability bound

$$
E n_{j}^{N}+\Delta t \sum_{n=0}^{N-1}\left\|\nabla u_{j, h}^{n}\right\|^{2} \leq C\left(E n_{j}^{0}+\sum_{n=0}^{N-1} \frac{\Delta t}{2 \nu_{j}}\left\|f_{j}\left(t^{n+1}\right)\right\|_{-1}^{2}\right)
$$

if we assume the following, slightly restrictive timestep condition

$$
\frac{2 \Delta t}{\nu_{j}}\left(\left\|u_{j, h}^{n^{\prime}}\right\|_{\infty}+\frac{\operatorname{diam}(\Omega)}{d}\left\|\nabla \cdot u_{j, h}^{n^{\prime}}\right\|_{\infty}\right)^{2} \leq 1, \forall j=1, \ldots, J,
$$

4.2 Stability with outflow boundary conditions

Now we consider the case of $\Gamma_{N} \neq \emptyset$. The stability for the Algorithm 2 holds under the following two timestep conditions

$$
\begin{aligned}
& \frac{\Delta t}{\nu_{j}}\left(\left\|u_{j, h}^{n^{\prime}}\right\|_{\infty}+\right.\left.\frac{1}{\sqrt{\lambda_{1}}}\left\|\nabla \cdot u_{j, h}^{n^{\prime}}\right\|_{\infty}\right)^{2} \leq 1, \forall j=1, \ldots, J, \\
& \frac{\Delta t}{8 \nu_{j}}\left\|u_{j, h}^{n^{\prime}} \cdot \mathbf{n}\right\|_{\infty, \Gamma_{N}}^{2} \leq 1, \forall j=1, \ldots, J
\end{aligned}
$$

where $\lambda_{1}>0$ is the smallest eigenvalue of the mixed Dirichet-Neummann spectral problem

$$
\begin{aligned}
-\Delta u & =\lambda u \text { in } \Omega, \\
u & =0 \text { on } \Gamma_{D}, \\
\frac{\partial u}{\partial \mathbf{n}} & =0 \text { on } \Gamma_{N} .
\end{aligned}
$$

\section{Theorem 41 Let}

$$
E n_{j}^{n}:=\frac{\left\|u_{j, h}^{n}\right\|^{2}+\mathrm{L}\left\|u_{j, h}^{n}\right\|_{\Gamma_{N}}^{2}}{2}+\Delta t \frac{\nu_{\infty}}{2}\left\|\nabla u_{j, h}^{n}\right\|^{2}
$$

If for each time step $n \geq 1$, the conditions (34)-(35) hold, then the solutions to Algorithm 2 satisfy

$$
\begin{aligned}
E n_{j}^{N} & +\Delta t \sum_{n=0}^{N-1} \int_{\Gamma_{N}}\left[\frac{\overline{u_{h}^{n}} \cdot \mathbf{n}}{2}\left|u_{j, h}^{n+1}\right|^{2} \Theta_{1}\left(\overline{u_{h}^{n}} \cdot \mathbf{n}\right)+\frac{u_{j, h}^{n^{\prime}} \cdot \mathbf{n}}{2}\left|u_{j, h}^{n}\right|^{2} \Theta_{1}\left(u_{j, h}^{n^{\prime}} \cdot \mathbf{n}\right)\right] \\
& \leq \exp \left(\frac{\nu_{j} T}{\mathrm{~L}}\right)\left(E n_{j}^{0}+\sum_{n=0}^{N-1} \frac{\Delta t}{2 \nu_{j}}\left\|f_{j}\left(t^{n+1}\right)\right\|_{*, D}^{2}\right) .
\end{aligned}
$$


Proof Choose the test functions $v_{h}=u_{j, h}^{n+1}, q_{h}=p_{j, h}^{n+1}$, and add the equations (14)-(15). The first nonlinear term becomes

$$
\begin{aligned}
b_{1}\left(\overline{u_{h}^{n}}, u_{j, h}^{n+1}, u_{j, h}^{n+1}\right)+ & b_{1}\left(u_{j, h}^{n^{\prime}}, u_{j, h}^{n}, u_{j, h}^{n+1}\right) \\
& =b_{1}\left(\overline{u_{h}^{n}}, u_{j, h}^{n+1}, u_{j, h}^{n+1}\right)+b_{1}\left(u_{j, h}^{n^{\prime}}, u_{j, h}^{n}, u_{j, h}^{n}\right) \\
& +b_{1}\left(u_{j, h}^{n^{\prime}}, u_{j, h}^{n}, u_{j, h}^{n+1}-u_{j, h}^{n}\right) \\
& =\int_{\Gamma_{N}}\left[\frac{\overline{u_{h}^{n}} \cdot \mathbf{n}}{2}\left|u_{j, h}^{n+1}\right|^{2}+\frac{u_{j, h}^{n^{\prime}} \cdot \mathbf{n}}{2}\left|u_{j, h}^{n}\right|^{2}\right] \\
& +b_{1}\left(u_{j, h}^{n^{\prime}}, u_{j, h}^{n}, u_{j, h}^{n+1}-u_{j, h}^{n}\right),
\end{aligned}
$$

and similarly,

$$
\begin{aligned}
b_{2}\left(\overline{u_{h}^{n}}, u_{j, h}^{n+1}, u_{j, h}^{n+1}\right) & +b_{2}\left(u_{j, h}^{n^{\prime}}, u_{j, h}^{n}, u_{j, h}^{n+1}\right) \\
& =-\int_{\Gamma_{N}}\left[\frac{\overline{u_{h}^{n}} \cdot \mathbf{n}}{2}\left|u_{j, h}^{n+1}\right|^{2} \Theta_{0}\left(\overline{u_{h}^{n}} \cdot \mathbf{n}\right)+\frac{u_{j, h}^{n^{\prime}} \cdot \mathbf{n}}{2}\left|u_{j, h}^{n}\right|^{2} \Theta_{0}\left(u_{j, h}^{n^{\prime}} \cdot \mathbf{n}\right)\right] \\
& +b_{2}\left(u_{j, h}^{n^{\prime}}, u_{j, h}^{n}, u_{j, h}^{n+1}-u_{j, h}^{n}\right) .
\end{aligned}
$$

Taking (38)-(39) into account gives

$$
\begin{gathered}
\frac{\left\|u_{j, h}^{n+1}\right\|^{2}-\left\|u_{j, h}^{n}\right\|^{2}+\left\|u_{j, h}^{n+1}-u_{j, h}^{n}\right\|^{2}}{2 \Delta t}+\mathrm{L} \frac{\left\|u_{j, h}^{n+1}\right\|_{\Gamma_{N}}^{2}-\left\|u_{j, h}^{n}\right\|_{\Gamma_{N}}^{2}+\left\|u_{j, h}^{n+1}-u_{j, h}^{n}\right\|_{\Gamma_{N}}^{2}}{2 \Delta t} \\
+\nu_{\infty}\left\|\nabla u_{j, h}^{n+1}\right\|^{2}+\underbrace{\int \frac{u_{h}^{n}}{\Gamma_{N}}\left[\frac{\mathbf{n}}{2}\left|u_{j, h}^{n+1}\right|^{2} \Theta_{1}\left(\overline{u_{h}^{n}} \cdot \mathbf{n}\right)+\frac{u_{j, h}^{n^{\prime}} \cdot \mathbf{n}}{2}\left|u_{j, h}^{n}\right|^{2} \Theta_{1}\left(u_{j, h}^{n^{\prime}} \cdot \mathbf{n}\right)\right]}_{:=F_{n+1} \geq 0} \\
=\left\langle f_{j}\left(t^{n+1}\right), u_{j, h}^{n+1}\right\rangle+\left(\nu_{\infty}-\nu_{j}\right)\left(\nabla u_{j, h}^{n}, \nabla u_{j, h}^{n+1}\right)-\sum_{i=1}^{2} b_{i}\left(u_{j, h}^{\left.n^{\prime}, u_{j, h}^{n}, u_{j, h}^{n+1}-u_{j, h}^{n}\right)} .\right.
\end{gathered}
$$

The first two terms on the right hand side and $b_{1}\left(u_{j, h}^{n^{\prime}}, u_{j, h}^{n}, u_{j, h}^{n+1}-u_{j, h}^{n}\right)$ are treated as in the proof of Theorem 7 . As for the $b_{2}\left(u_{j, h}^{n^{\prime}}, u_{j, h}^{n}, u_{j, h}^{n+1}-u_{j, h}^{n}\right)$, we apply Cauchy-Schwarz to get

$$
\begin{aligned}
b_{2}\left(u_{j, h}^{n^{\prime}}, u_{j, h}^{n}, u_{j, h}^{n+1}-u_{j, h}^{n}\right) & =\int_{\Gamma_{N}} \frac{-u_{j, h}^{n^{\prime}} \cdot \mathbf{n}}{2} u_{j, h}^{n} \cdot\left(u_{j, h}^{n+1}-u_{j, h}^{n}\right) \Theta_{0}\left(u_{j, h}^{n^{\prime}} \cdot \mathbf{n}\right) \\
& \leq \mathrm{L} \frac{\left\|u_{j, h}^{n+1}-u_{j, h}^{n}\right\|_{\Gamma_{N}}^{2}}{2 \Delta t}+\frac{\Delta t\left\|u_{j, h}^{n^{\prime}} \cdot \mathbf{n}\right\|_{\infty, \Gamma_{N}}^{2}}{8 \mathrm{~L}}\left\|u_{j, h}^{n}\right\|_{\Gamma_{N}}^{2} \\
& \leq \mathrm{L} \frac{\left\|u_{j, h}^{n+1}-u_{j, h}^{n}\right\|_{\Gamma_{N}}^{2}}{2 \Delta t}+\frac{\nu_{j}}{\mathrm{~L}}\left\|u_{j, h}^{n}\right\|_{\Gamma_{N}}^{2} .
\end{aligned}
$$

The last bounded has been obtained under (35). Putting everything together and summing over the timesteps yields

$$
E n_{j}^{N}+\Delta t \sum_{n=1}^{N-1} F_{n+1} \leq E n_{j}^{0}+\frac{\nu_{j}}{\mathrm{~L}} \Delta t \sum_{n=1}^{N-1}\left\|u_{j, h}^{n}\right\|_{\Gamma_{N}}^{2}+\frac{\Delta t}{2 \nu_{j}} \sum_{n=1}^{N-1}\left\|f_{j}^{n+1}\right\|_{*, D}^{2}
$$

Gronwall's inequality completes the proof. 
Now we prove the stability of the Algorithm 3 under

$$
\frac{C \Delta t}{h \nu_{j}}\left\|\nabla u_{j, h}^{n^{\prime}}\right\|^{2} \leq 1, \forall j=1, \ldots, J, C=\mathcal{O}(1)
$$

Theorem 42 Let

$$
E n_{j}^{n}:=\frac{\left\|u_{j, h}^{n}\right\|^{2}}{2}+\Delta t \frac{\nu_{\infty}}{2}\left\|\nabla u_{j, h}^{n}\right\|^{2} .
$$

If for each time step $n \geq 1$, the condition (43) holds, then the solutions to Algorithm 3 satisfy

$$
\begin{aligned}
E n_{j}^{N} & +\Delta t \sum_{n=0}^{N-1} \int_{\Gamma_{N}} \frac{\overline{u_{h}^{n}} \cdot \mathbf{n}}{2}\left|u_{j, h}^{n+1}\right|^{2} \Theta_{1}\left(\overline{u_{h}^{n}} \cdot \mathbf{n}\right) \\
& \leq E n_{j}^{0}+\sum_{n=0}^{N-1} \frac{\Delta t}{2 \nu_{j}}\left\|f_{j}\left(t^{n+1}\right)\right\|_{*, D}^{2} .
\end{aligned}
$$

Proof The proof is very similar to that of Theorems 7-41. The only difference is bounding the $b_{3}(\cdot, \cdot, \cdot)$ term:

$$
\begin{aligned}
b_{3}\left(u_{j, h}^{n^{\prime}}, u_{j, h}^{n}, u_{j, h}^{n+1}\right) & =b_{3}\left(u_{j, h}^{n^{\prime}}, u_{j, h}^{n}, u_{j, h}^{n+1}-u_{j, h}^{n}\right) \\
& \leq C\left\|\nabla u_{j, h}^{n^{\prime}}\right\|\left\|\nabla u_{j, h}^{n}\right\| \sqrt{\left\|\nabla\left(u_{j, h}^{n+1}-u_{j, h}^{n}\right)\right\|\left\|u_{j, h}^{n+1}-u_{j, h}^{n}\right\|} \\
& \leq C h^{-1 / 2}\left\|\nabla u_{j, h}^{n^{\prime}}\right\|\left\|\nabla u_{j, h}^{n}\right\|\left\|u_{j, h}^{n+1}-u_{j, h}^{n}\right\| \\
& \leq \frac{\left\|u_{j, h}^{n+1}-u_{j, h}^{n}\right\|^{2}}{2 \Delta t}+C \frac{\Delta t}{h}\left\|\nabla u_{j, h}^{n}\right\|^{2}\left\|\nabla u_{j, h}^{n^{\prime}}\right\|^{2} .
\end{aligned}
$$

4.3 Convergence with homogeneous Dirichlet boundary conditions

Convergence for the case of $\nu_{j}=\nu$ has been already been considered in the literature [9], and the effect of additional viscous terms are straightforward to analyze.

Theorem 8 Let $\left(X_{h}, Q_{h}\right)=\left(P_{2}, P_{1}\right)$, be a Taylor-Hood pair. Assuming enough smoothness on the exact solution and the timestep condition (33), the velocity error $e_{j}^{n}:=u_{j}\left(x, t^{n}\right)-u_{j, h}^{n}$ in the Algorithm 4 satisfies the following error estimate:

$$
\left\|e_{j}^{N}\right\|^{2}+\nu_{\infty} \Delta t \sum_{n=1}^{N}\left\|\nabla e_{j}^{n}\right\|^{2} \leq C\left(T, \nu_{\infty}-\nu_{j}\right)\left(h^{4}+\Delta t^{4}\right)
$$

\section{Numerical Experiments}

The simulations are performed using the FreeFem $++[17]$ package, with the $\left(P_{2}, P_{1}\right)$ used to approximate the velocity and pressure spaces, respectively. We only tested second order schemes. For the CFL conditions, we use the respective conditions for the first order schemes, replacing $u_{j, h}^{n^{\prime}}$ with $E_{j, h}^{n^{\prime}}$. All the linear systems are solved using direct solvers.

In the last two channel flow examples, for Algorithm 5, we tested few different values of $\mathrm{L}$. Namely, we set $\mathrm{L}=\tau \mathrm{D}$, where $\tau \leq 1$, and $\mathrm{D}$ is the inlet diameter of a channel. Larger values of $\tau$ altered the solution qualitatively near the outlet, as was also observed in [15], and therefore we used $\tau=0.01$ in both cases. 
Table 1: Velocity errors in $L^{2}$ norm for Algorithm 4

\begin{tabular}{|c|c|c|c|c|c|c|c|c|}
\hline$\Delta t$ & $\left\|u_{1}-u_{1, h}\right\|$ & rate & $\left\|u_{1}-\tilde{u}_{1, h}\right\|$ & rate & $\left\|u_{2}-u_{2, h}\right\|$ & rate & $\left\|u_{2}-\tilde{u}_{2, h}\right\|$ & rate \\
\hline 0.05 & $3.07772 e-06$ & 2.1518 & $3.06419 e-06$ & 2.1537 & $3.00379 e-06$ & 2.1544 & $3.0171 e-06$ & 2.1523 \\
\hline 0.025 & $6.92603 e-07$ & 2.0827 & $6.88645 e-07$ & 2.0838 & $6.74753 e-07$ & 2.0842 & $6.78692 e-07$ & 2.0830 \\
\hline 0.0125 & $1.63507 e-07$ & 2.0433 & $1.62446 e-07$ & 2.0439 & $1.59128 e-07$ & 2.0441 & $1.60186 e-07$ & 2.0434 \\
\hline 0.00625 & $3.96689 e-08$ & 2.0222 & $3.93949 e-08$ & 2.0225 & $3.8585 e-08$ & 2.0226 & $3.88584 e-08$ & 2.0223 \\
\hline 0.003125 & $9.76599 e-09$ & & $9.69639 e-09$ & & $9.49636 e-09$ & & $9.56583 e-09$ & \\
\hline
\end{tabular}

Table 2: Pressure errors in $L^{2}$ norm for Algorithm 4

\begin{tabular}{|c|c|c|c|c|c|c|c|c|}
\hline$\Delta t$ & $\left\|p_{1}-p_{1, h}\right\|$ & rate & $\left\|p_{1}-\tilde{p}_{1, h}\right\|$ & rate & $\left\|p_{2}-p_{2, h}\right\|$ & rate & $\left\|p_{2}-\tilde{p}_{2, h}\right\|$ & rate \\
\hline 0.05 & 0.000655589 & 2.0140 & 0.000657382 & 2.0138 & 0.000635395 & 2.0138 & 0.000633635 & 2.0139 \\
\hline 0.025 & 0.000162317 & 2.0071 & 0.000162776 & 2.0071 & 0.000157337 & 2.0070 & 0.000156888 & 2.0071 \\
\hline 0.0125 & $4.03813 e-05$ & 1.9953 & $4.04929 e-05$ & 1.9948 & $3.9145 e-05$ & 1.9998 & $3.90282 e-05$ & 1.9868 \\
\hline 0.00625 & $1.01284 e-05$ & 1.9988 & $1.01598 e-05$ & 1.9533 & $9.78791 e-06$ & 1.9859 & $9.84643 e-06$ & 2.0188 \\
\hline 0.003125 & $2.53421 e-06$ & & $2.62347 e-06$ & & $2.47108 e-06$ & & $2.42973 e-06$ & \\
\hline
\end{tabular}

\subsection{Convergence study}

We first confirm the predicted convergence rates, and also compare the accuracy of our scheme to independent simulations. For this problem, we take domain $\Omega=(0,1)^{2}$, viscosity $\nu=1$ and final time $T=1$. We generate perturbations using

$$
u=\left(\begin{array}{c}
x^{2}-y \sin (t) \\
-2 x y+x \cos (t)
\end{array}\right), p=(x+y-1) \sin (t) .
$$

Picking the perturbation parameter $\varepsilon=10^{-2}$, we consider two Navier-Stokes equations, whose solutions and source terms are

$$
u_{1,2}=(1 \pm \varepsilon) u, p_{1,2}=(1 \pm \varepsilon) p, f_{j}=\partial_{t} u_{j}+u_{j} \cdot \nabla u_{j}-\nu_{j} \Delta u_{j}+\nabla p_{j}, j=1,2,
$$

with $\nu_{j}=(1 \pm \varepsilon) \nu$.

Since the solutions are exact in space, the dominant source of the error will be temporal. Here we fixed the mesh size $h=\frac{1}{15}$ and refine the time step $\Delta t$. The errors are reported in the Table 1 and 2, which show the expected second order convergence rate, and almost the same accuracy between ensemble and sequential methods. In the tables, the tilde notation refers to independent simulations.

\subsection{Flow around a cylinder}

We test our Algorithms 5-6 on a two dimensional channel flow around a cylinder, a well-known benchmark problem taken from Shäfer and Turek [18]. The flow patterns are driven by the interaction of a fluid with a wall which is an important scenario for many industrial flows. The domain for the problem is a $2.2 \times 0.41$ rectangular channel with a cylinder of radius 0.05 centered at $(0.2,0.2)$ (taking the bottom left corner of the rectangle as the origin). The cylinder, top and bottom of the channel are prescribed no slip boundary conditions, and the time dependent inflow and outflow profile are

$$
\begin{aligned}
& u_{1}(0, y, t)=u_{1}(2.2, y, t)=\frac{6}{0.41^{2}} \sin (\pi t / 8) y(0.41-y) \\
& u_{2}(0, y, t)=u_{2}(2.2, y, t)=0
\end{aligned}
$$

The quantative results for this problem with $\nu=\frac{1}{1000}$ are given in [10] and [11] under Dirichlet outflow and do-nothing outflow conditions, respectively. Here we chose three ensemble members with viscosities as $\nu_{1}=\frac{1}{1000}, \nu_{2}=\frac{1}{900}, \nu_{3}=\frac{1}{800}$ and compare the results of the $\nu_{1}$ case with the reference values. The mesh used in the simulations is shown in Fig. 1 with diameter $h=0.0216741$. The smallest eigenvalue of the Dirichet-Neumann problem (36) is computed 


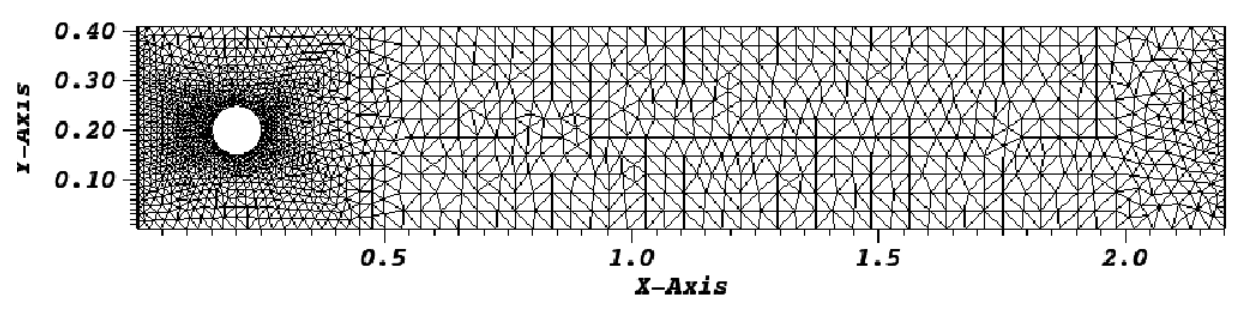

Fig. 1: The finite element mesh used in flow around a cylinder experiment. Number of elements is 3306

Table 3: Drag, Lift and pressure drop values

\begin{tabular}{|c|c|c|c|c|c|}
\hline method & $c_{d, \max }$ & $t\left(c_{d, \max }\right)$ & $c_{l, \max }$ & $t\left(c_{l, \max }\right)$ & $\Delta p$ \\
\hline Algorithm 5 & 2.9071 & 3.9400 & 0.4754 & 5.6820 & -0.1140 \\
\hline Algorithm 6 & 2.9071 & 3.9240 & 0.4754 & 5.6820 & -0.1140 \\
\hline (Dirichlet) [10] & 2.95092 & 3.93625 & 0.47795 & 5.69313 & -0.1116 \\
\hline (No-traction) [11] & 2.9513 & 4.0112 & 0.47887 & 5.6928 & -0.026382 \\
\hline
\end{tabular}

to be $\lambda_{1}=59.3467$. We started all cases with the time step $\Delta t=0.004$. Stability is checked according to the inequalities (34)-(35) for the Algorithm 5, and (43) for the Algorithm 6. If it is violated, the time step is halved. In our simulations, the final value of $\Delta t$ was 0.001 for both algorithms. We compute values for the maximal drag $c_{d, \max }$ and lift $c_{l, \max }$ coefficients on the cylinder boundary, and the pressure difference $\Delta p(t)$ between the front and back of the cylinder at the final time $T=8$. The time evolutions of the these quantities are in Fig. 2 .

The maximum lift and drag coefficients and pressure drop for the simulations are given in Table 3, and we see that our algorithm performs well. The velocity contour plots at times $\mathrm{t}=$ 6, 8 are presented in Figs. 3 and 4, and streamlines are given in those plots to show a vortex street. Qualitatively, the plots match the reference plots from [11], and those two algorithms 5 and 6 gave the same results. We compare Figs. 3 and 4 with the results obtained using the open boundary or zero traction boundary conditions in [11]. With our method, at $t=8$ the last eddy is cut through by the outflow boundary $x=2.2$. This agrees with the results in [11] unlike giving a prescribed parabolic velocity profile where the last eddy will remain on the left hand side of $x=2.2$ completely, as in [10]. The prescribed Dirichlet type parabolic outflow profile is less physical because following the previous alternating pattern from upstream, it is unrealistic that both eddies near the top and bottom walls will vanish at the same position at $x=2.2$.

\subsection{Channel flow with a contraction and two outlets}

Our last experiment is for a complex 2-d flow through a channel with a contraction and two outlets, one on the top of the channel and the other one is at the end of the channel. Mesh is shown in Fig. 5

We again consider the case of three ensemble members. We run the simulations on time interval $(0,4)$, with $\nu_{1}=0.001, \nu_{2}=0.003$ and $\nu_{3}=0.005,\left(X_{h}, Q_{h}\right)=\left(P_{2}, P_{1}\right)$ TaylorHood finite element pair. The velocity boundary conditions are: no-slip on the walls, $g_{1}=$ $(4 y(1-y), 0)^{T}, g_{2}=(1+\varepsilon)(4 y(1-y), 0)^{T}, g_{3}=(1-\varepsilon)(4 y(1-y), 0)^{T}$ at the inlet, and open boundary condition at the outlets. Here we test our Algorithm 5 with $\mathrm{L}=0.01$ and Algorithm 6. Initial conditions $u_{1, h}^{0}, u_{2, h}^{0}, u_{3, h}^{0}$ are obtained by solving Stokes equations in the same domain with perturbed body forces $f_{1}=\varepsilon(0,0)^{T}, f_{2}=\varepsilon(\cos (\pi x y+t), \sin (\pi(x+y)+t))^{T}$ and $f_{3}=\varepsilon(\sin (\pi(x+y)+t), \cos (\pi x y+t))^{T}$ with $\varepsilon=10^{-2}$.

We ran both ensemble and independent runs. In the ensemble runs, we start with $\Delta t=0.01$, and half the time step once the stability condition inequalities (34)-(35) with $\lambda_{1}=11.8335$ is violated. For Algorithm 5, the time step was halved once and the final time step is $\Delta t=$ 

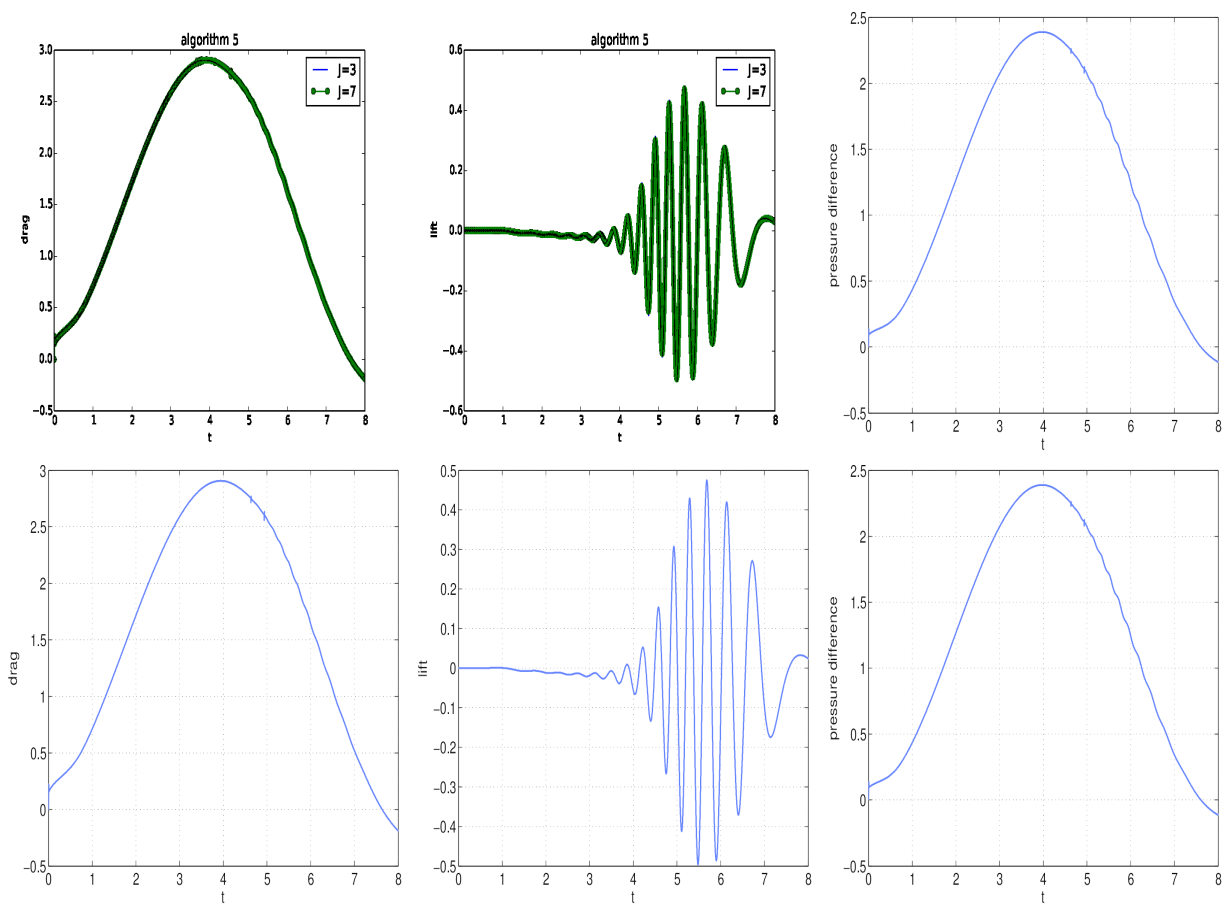

Fig. 2: From left to right: the drag and lift coefficients $c_{d}, c_{l}$ and pressure difference between front and back of the cylinder $\Delta p$ for flow past a cylinder with Algorithm 5 (top) and Algorithm 6 (bottom)
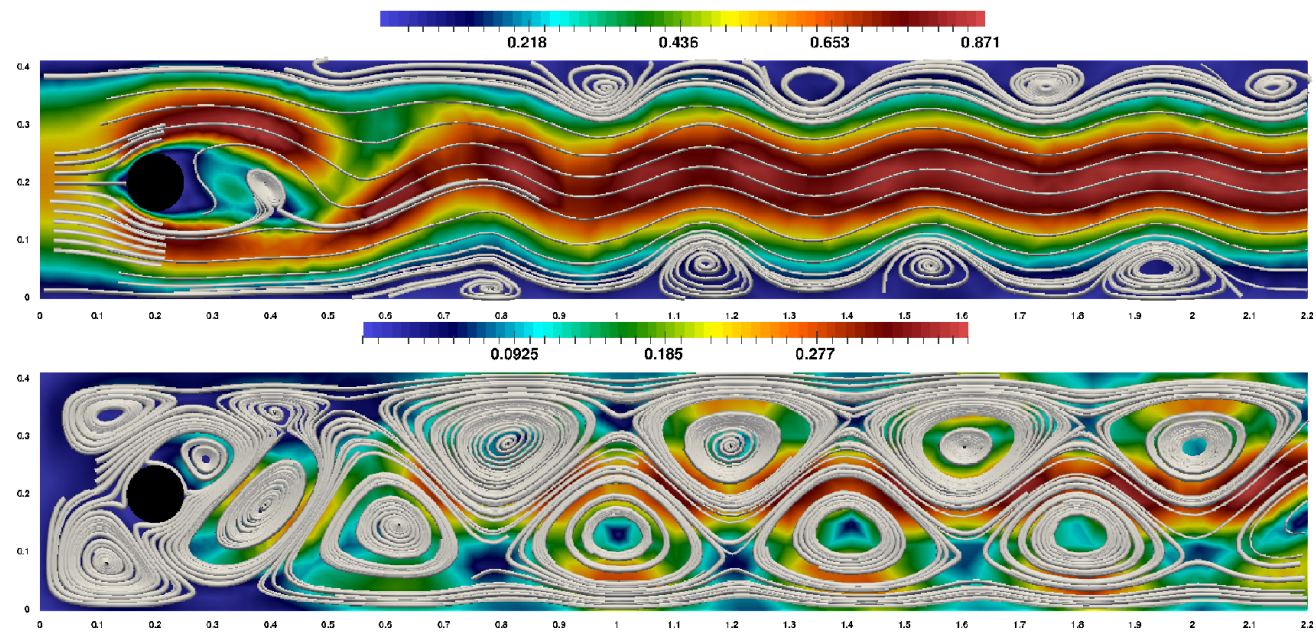

Fig. 3: Velocity field at $\mathrm{t}=6,8$, with algorithm 5

0.005. For Algorithm 6, the time step was refined a few times and the final time step is $\Delta t=0.0003125$.

Simulations are performed on a mesh with 16,672 DOF. Since the simulations on this mesh are underresolved, we use the adaptive nonlinear filter scheme of [23] to stabilize the solutions. As a reference, we also performed independent DNS runs for $\nu_{2}$ and $\nu_{3}$ on a mesh with total of 290, 000 DOF and second order timestepping scheme. Due to the computational cost, we only ran the DNS simulations till $T=1$ and compare speed contours with Algorithm 5 in Fig. 6 and Algorithm 6 in Fig. 7. We can observe that, the ensemble scheme gives qualitatively same results as independent simulations. The speed contour for $\nu_{1}=0.001$ is shown for ensemble 


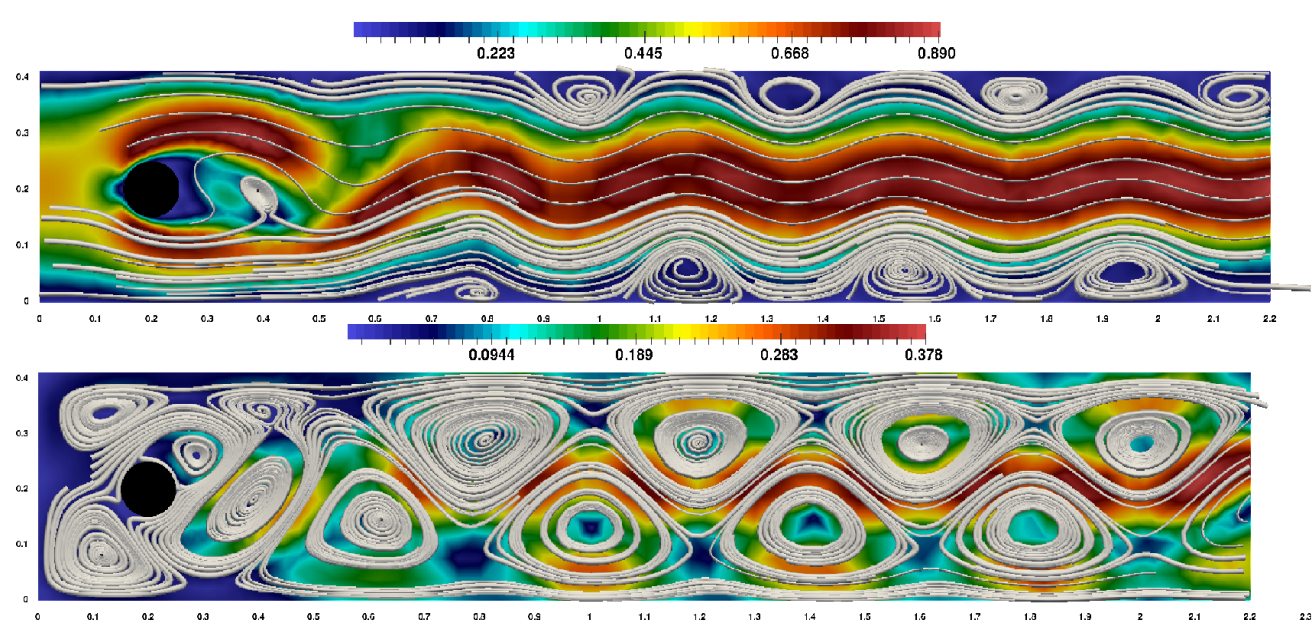

Fig. 4: Velocity field at $\mathrm{t}=6,8$, with algorithm 6

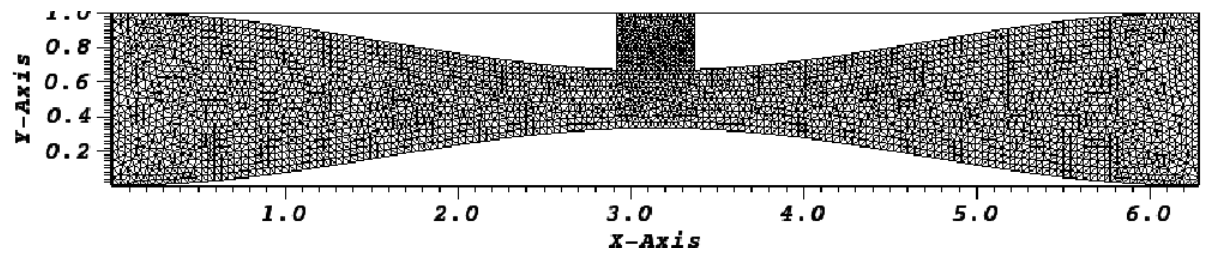

Fig. 5: The finite element mesh used in Channel flow with a contraction and two outlets. DOF is 16,672 .

method and independent runs. Notice that in the ensemble method, for member $\nu_{1}=0.001$, no perturbation is added in order to get a fair comparison. In Fig. 8 Algorithm 5 is used for the ensemble method and corresponding sequential run is given here as well and Fig. 9 is with Algorithm 6, and they gave very similar results.

\section{Conclusions}

We revisited the algorithm of [8], and proposed a new one with better stability properties. We also developed first and second order ensemble schemes for open boundary conditions, with provable stability bounds. The numerical tests at moderate Re number show that the ensemble simulation match the independent simulation results, both qualitatively and quantatively.

We believe that the ensemble schemes need further testing and research in order to fully understand their advantages and possible disadvantages. One of the projects we will undertake in the future is the CPU time comparison of an ensemble scheme against fully explicit scheme on adaptively refined meshes for high Re number flows.

\section{References}

1. Pahlevani, Faranak. Sensitivity computations of eddy viscosity models with an application in drag computation, Int. J. Num. Meth. Fluids, 52(4), pp. 381-392, 2016.

2. Sagaut, Pierre and Lê, Thien Hiep. Some Investigations on the Sensitivity of Large Eddy Simulation, in Direct and Large-Eddy Simulation II, 1997, pp. 81-92.

3. Burkardt, John and Gunzburger, Max and Lee, Hyung-Chun. POD and CVT-based reduced-order modeling of Navier?Stokes flows, Comp. Meth. Appl. Mech. Eng., 196(1), pp. 337-355, 2006.

4. Howard, Clint and Gupta, Sushen and Abbas, Ali and A.G. Langrish, Timothy and F. Fletcher, David. Proper Orthogonal Decomposition (POD) analysis of CFD data for flow in an axisymmetric sudden expansion, Chem. Eng. Res. Des, 123, pp. 333 - 346, 2017.

5. S. Walton and O. Hassan and K. Morgan. Reduced order modelling for unsteady fluid flow using proper orthogonal decomposition and radial basis functions, Appl. Math. Model., 37(20), pp. 8930 - 8945, 2013. 

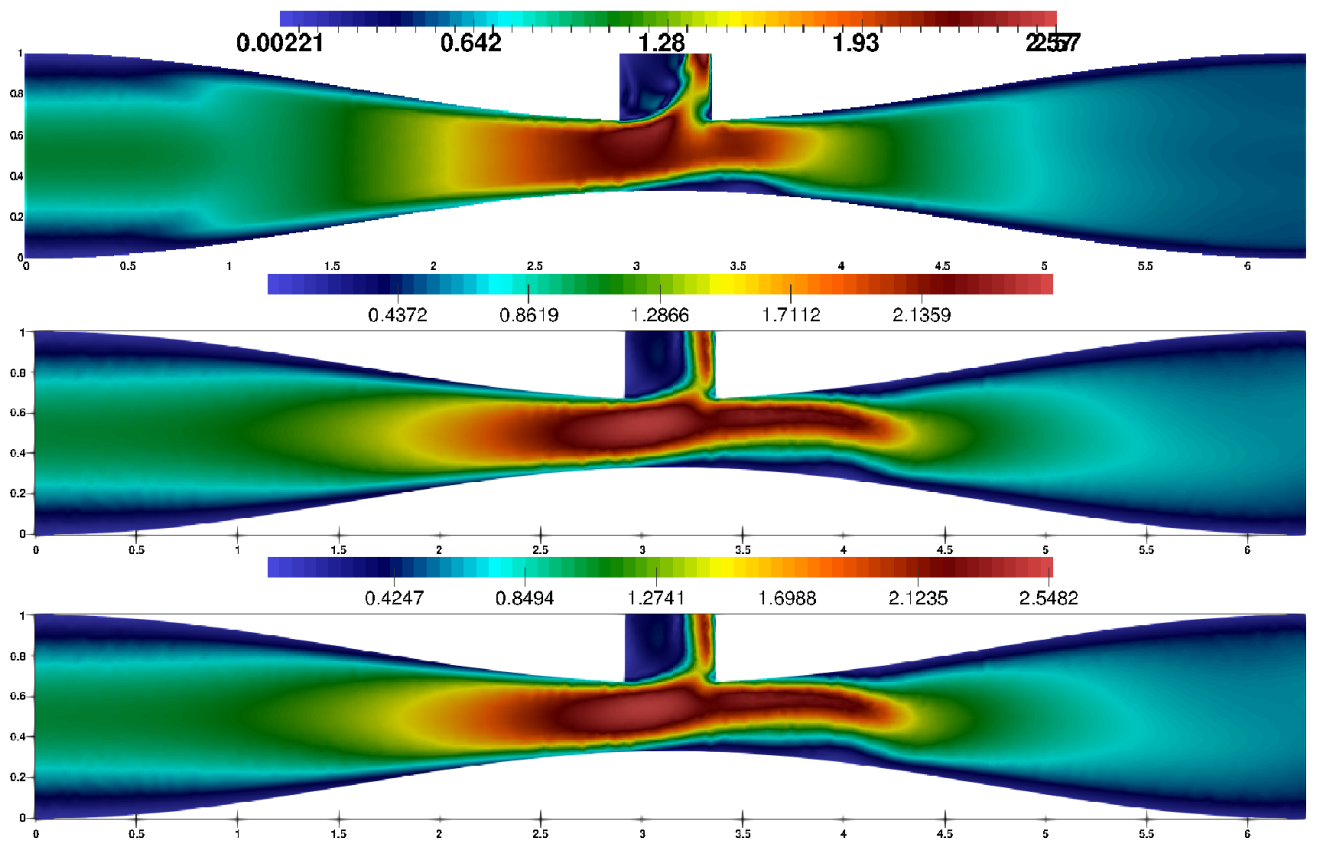

Fig. 6: Plots of velocity $u_{2}$ from DNS, ensemble simulation and independent simulation from top to bottom at $T=1$ with Algorithm 5 .
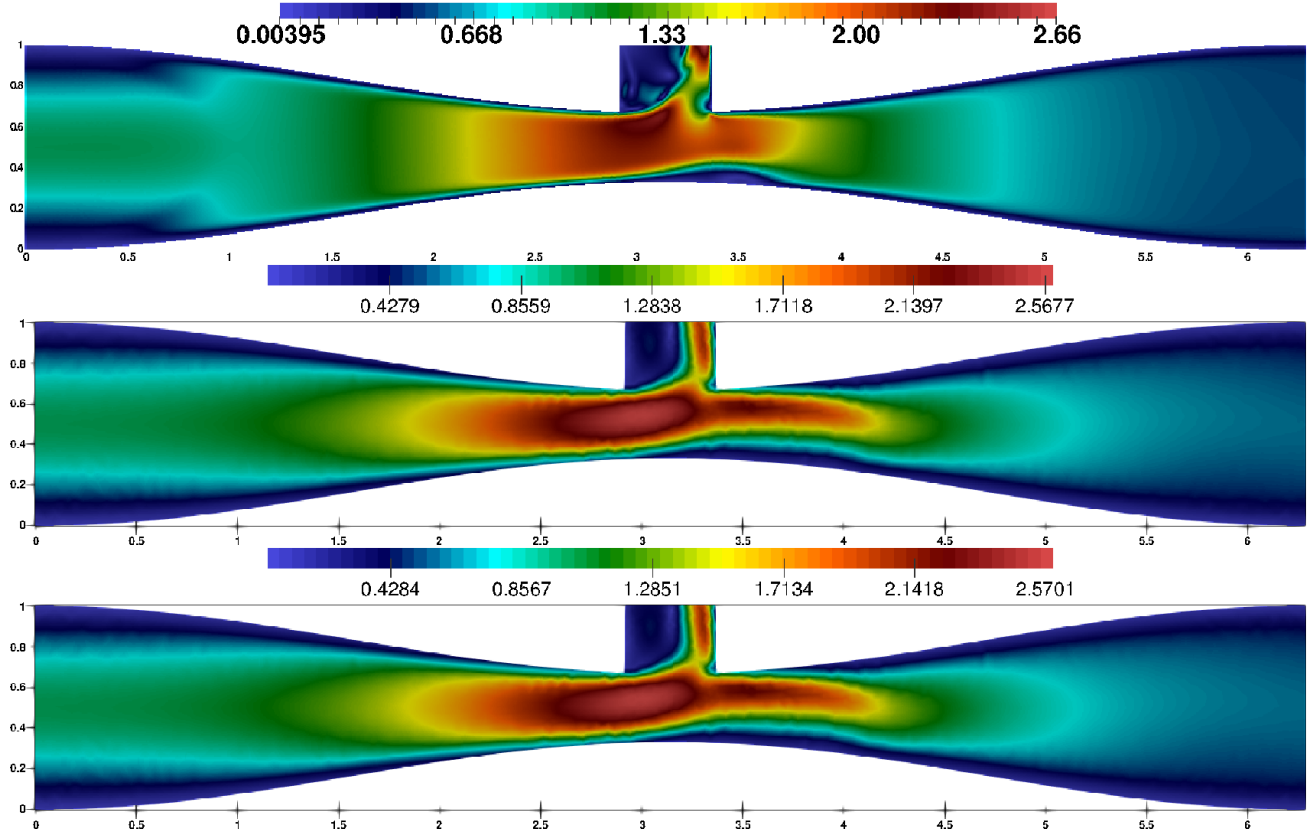

Fig. 7: Plots of velocity $u_{3}$ from DNS, ensemble simulation and independent simulation from top to bottom at $T=1$ with Algorithm 5 . 


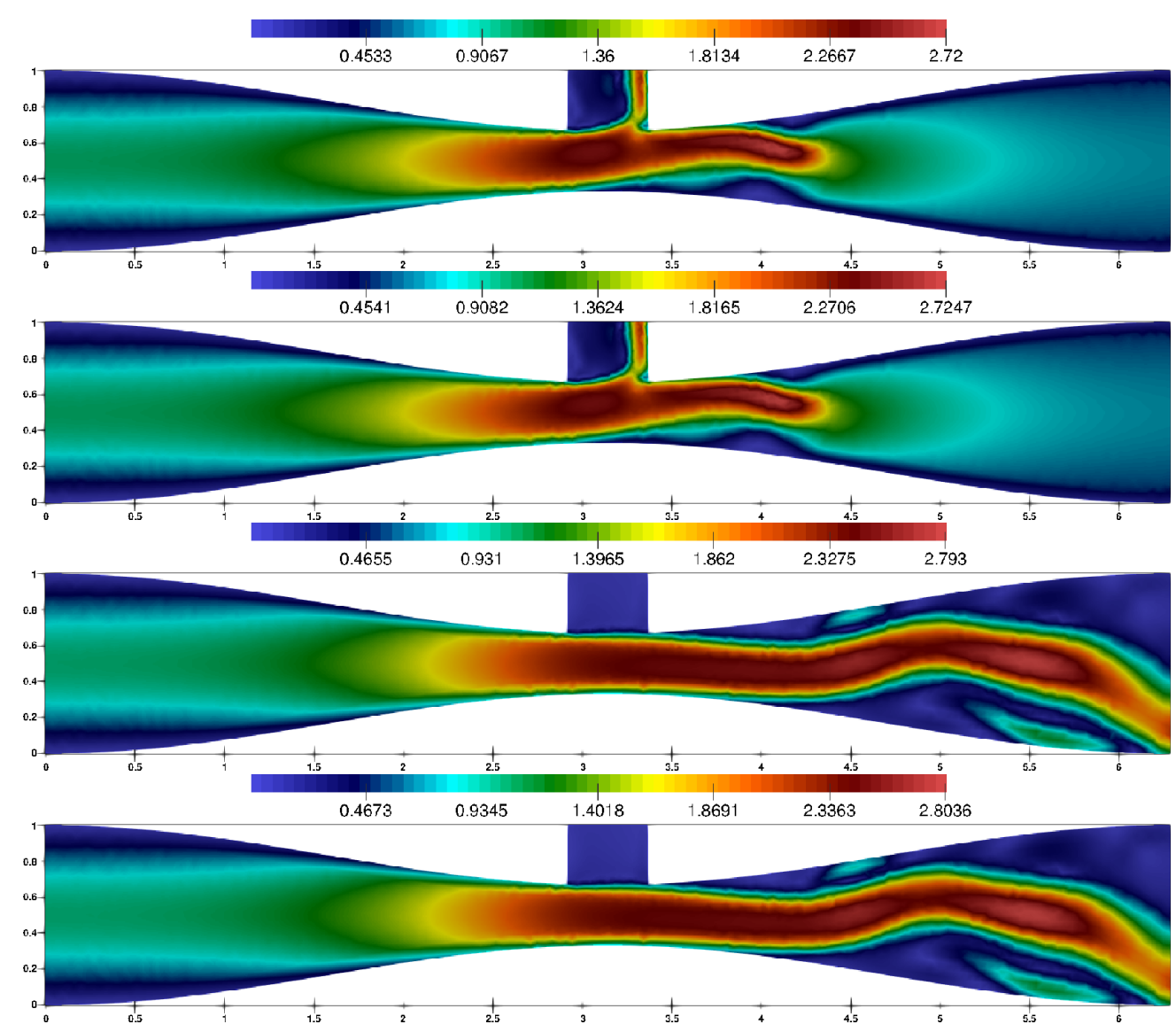

Fig. 8: Plots of velocity $u_{1}$ with Algorithm 5 simulation and independent simulation from top to bottom at $T=1$ (top 2 ) and $T=4$ (bottom 2).

6. Christensen, H. M. and Moroz, I. M. and Palmer, T. N. Stochastic and Perturbed Parameter Representations of Model Uncertainty in Convection Parameterization, 72(6), pp. 2525-2544, 2015.

7. Toth, Zoltan and Kalnay, Eugenia. Ensemble Forecasting at NCEP and the Breeding Method, Monthly Weather Review, 125(12), pp. 3297-3319, 1997.

8. Gunzburger, Max and Jiang, Nan and Wang, Zhu. An efficient algorithm for simulating ensembles of parameterized flow problems, IMA Journal of Numerical Analysis, 2018.

9. Nan Jiang and William Layton. An algorithm for fast calculation of flow ensembles. International Journal for Uncertainty Quantification, 2152-5080, 4 (4), 273-301, 2014.

10. John, V., Reference values for drag and lift of a two dimensional time-dependent flow around a cylinder, Int. J. Numer. Meth. Fluids, 44, pp. 777-788, 2004

11. Jie Liu. Open and traction boundary conditions for the incompressible Navier-Stokes equations, J. Comp. Phys., 228(19), pp. 7250 - 7267, 2009.

12. Jiang, Nan and Layton, William. Numerical analysis of two ensemble eddy viscosity numerical regularizations of fluid motion, Num. Meth. Part. Diff. Eq., 31(3), pp. 630-651, 2015.

13. Jiang and Nan, A Higher Order Ensemble Simulation Algorithm for Fluid Flows, J. Sci. Comp., 64(1), pp. 264-288, 2015.

14. Takhirov, Aziz and Neda, Monika and Waters, Jiajia. Time relaxation algorithm for flow ensembles, Num. Met. Part. Diff. Eq., 32(3), pp. 757-777, 2016.

15. S. Dong, A convective-like energy-stable open boundary condition for simulations of incompressible flows, J. Comp. Phys., 302, pp. 300-328, 2015.

16. Alfio Quarteroni, Numerical Models for Differential Problems, Springer-Verlag Milan, 2009.

17. Hecht, F., New development in FreeFem++, J. Numer. Math., 20(3-4), pp 251-265, 2012.

18. M. Schäfer and S. Turek, The benchmark problem 'flow around a cylinder' flow simulation with high performance computers II, in E.H. Hirschel (Ed.), Notes on Numerical Fluid Mechanics, 52, pp. 547-566, 1996.

19. K. Jbilou, Smoothing iterative block methods for linear systems with multiple right-hand sides, J. Comp. Appl. Math., 107(1), pp. 97-109, 1999.

20. Heyouni, M. and Essai, A., Matrix Krylov subspace methods for linear systems with multiple right-hand sides, Numerical Algorithms, 40(2), pp. 137-156, 2005. 

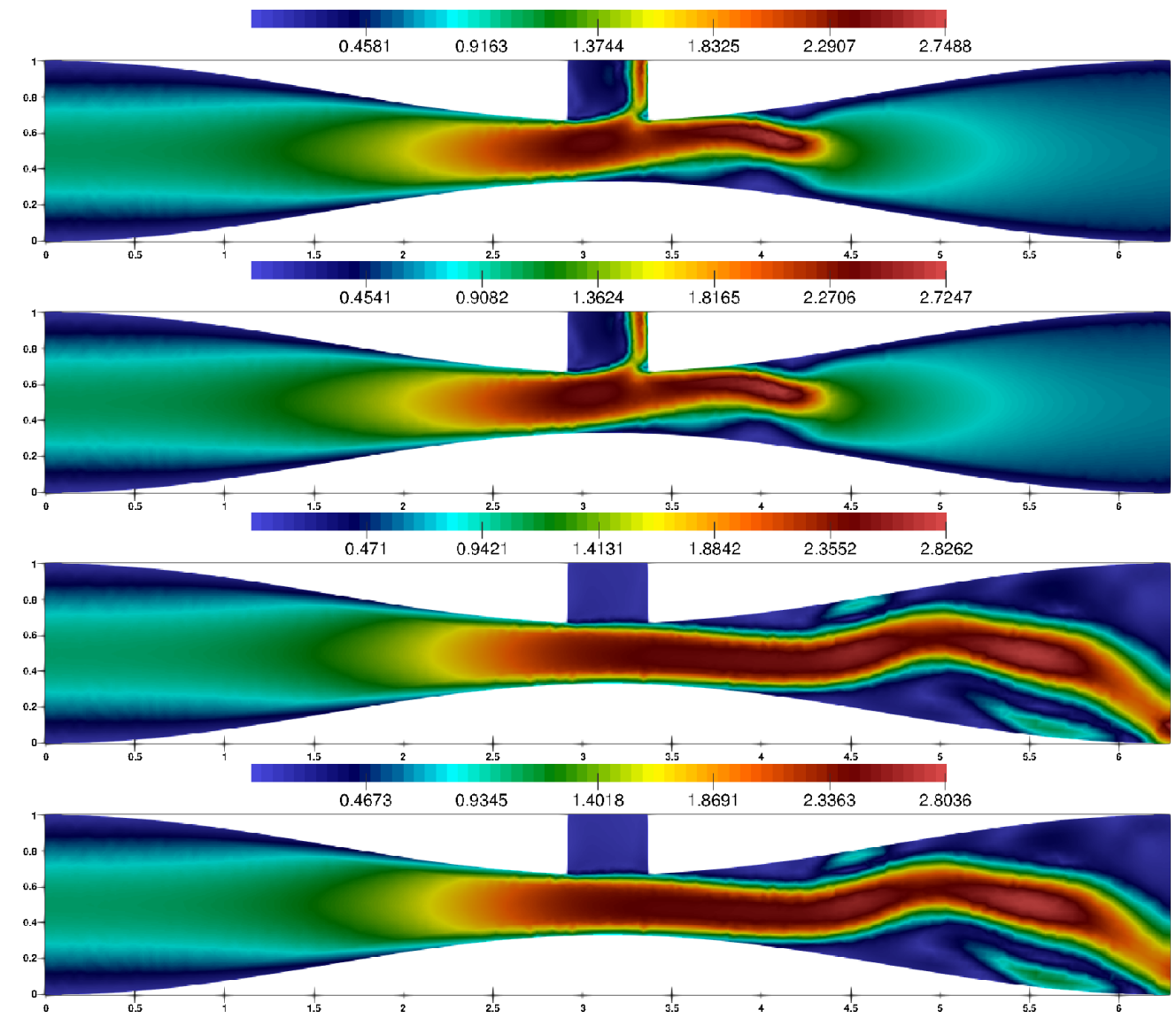

Fig. 9: Plots of velocity $u_{1}$ from Algorithm 6 simulation and independent simulation from top to bottom at $T=1$ (top 2 ) and $T=4$ (bottom 2 ).

21. Sergey Charnyi and Timo Heister and Maxim A. Olshanskii and Leo G. Rebholz On conservation laws of Navier-Stokes Galerkin discretizations, J. Comp. Phys., 337, pp. 289-308, 2017.

22. D.N. Arnold and J. Qin, Quadratic Velocity/Linear Pressure Stokes Elements, Advances in Computer Methods for Partial Differential Equations VII, IMACS, pp. 28-34, 1992.

23. Takhirov, Aziz and Lozovskiy, Alexander, Computationally efficient modular nonlinear filter stabilization for high Reynolds number flows, Adv. Comp. Math., 44(1), pp. 295-325, 2017. 